\title{
Enhanced photovoltaic performance of CdS-sensitized inverted organic solar cells prepared via a successive ionic layer adsorption and reaction method
}

\begin{abstract}
One-dimensional $\mathrm{ZnO}$ nanorods (ZNRs) synthesized on fluorine-doped tin oxide (FTO) glass by hydrothermal method were modified with cadmium sulfide quantum dots (CdS QDs) as an electron transport layer (ETL) in order to enhance the photovoltaic performance of inverted organic solar cell (IOSC). In present study, CdS QDs were deposited on ZNRs using a Successive Ionic Layer Adsorption and Reaction method (SILAR) method. In typical procedures, IOSCs were fabricated by spin-coating the P3HT:PC61BM photoactive layer onto the as-prepared ZNRs/CdS QDs. The results of current-voltage (I-V) measurement under illumination shows that the FTO/ZNRs/CdS QDs/ P3HT:PC61BM/ PEDOT: PSS/Ag IOSC achieved a higher power conversion efficiency $(4.06 \%)$ in comparison to FTO/ZNRs/P3HT:PC61BM/PEDOT: PSS/Ag (3.6\%). Our findings suggest that the improved open circuit voltage (Voc) and short circuit current density (Jsc) of ZNRs/CdS QDs devices could be attributed to enhanced electron selectivity and reduced interfacial charge carrier recombination between ZNRs and P3HT:PC61BM after the deposition of CdS QDs. The CdS QDs sensitized ZNRs reported herein exhibit great potential for advanced optoelectronic application.
\end{abstract}

Keyword: Inverted organic solar cells; ZNRs; CdS QDs; P3HT:PC61BM; SILAR 